\title{
Efficient micro-recovery and guanidination of peptides directly from MALDI target spots
}

\author{
Steven L. Cockrill, Kelly L. Foster, Justin Wildsmith, April R. Goodrich, John \\ G. Dapron, Tom C. Hassell, William K. Kappel, and Graham B.I. Scott \\ Sigma-Aldrich Corporation, St. Louis, MO, USA
}

BioTechniques 38:301-304 (February 2005)

\begin{abstract}
A method is presented for the recovery and subsequent guanidination of tryptic peptides from samples previously spotted on a matrix-assisted laser desorption/ionization (MALDI) target. The procedure is shown to have applicability to both in-solution and in-gel digests, yielding improved confidence in protein identification and sequence coverage in all instances. Recovery from the plate is essentially quantitative, with no residual analyte observed on the target spot. The technique is rapid, simple, and has extended applicability to other processing steps, including (but not limited to) derivatization for specific peptide studies or enzymatic treatment for subsequent profiling of posttranslational modifications. This method circumvents the failure of an initial analysis to generate suitable information and is particularly relevant for the analysis of precious samples.
\end{abstract}

\section{INTRODUCTION}

Enzymatic digestion of proteins with trypsin followed by analysis of the resulting peptides by mass spectrometry is a fundamental protocol in proteomics research. Trypsin specifically cleaves proteins on the carboxyl side of arginine and lysine residues (except where proline is C-terminal), thus generating peptides with either arginine or lysine as C-terminal amino acids.

Previous studies have suggested that the detection of peptides in mass spectrometric analysis is influenced by several intrinsic properties, including the basicity of the C-terminal amino acid (1-3), amino acid composition (2), peptide hydrophobicity and size (1), and the potential to form stable secondary structures (4). In addition, suppression effects have been described for peptide mixtures such as those obtained by tryptic digestion (5). Accordingly, sequence coverage and protein identification may be compromised. Furthermore, if the peptides of specific interest (such as those with posttranslational modifications) have lysine at the $\mathrm{C}$ terminus, the less favorable ionization of these peptides can be of critical concern in the acquisition of data. One method to overcome this issue is to convert the lysine residue to homoarginine through guanidination of the $\varepsilon$-amine side chain
(6-11). By reduction of the ionization bias, an overall increase in sequence coverage may be realized, with concomitant improvement in data confidence. The guanidination reaction has also been utilized to facilitate a number of different protein analysis methods. These include protein quantitation (12-14) and peptide sequencing through $\mathrm{N}$-terminal sulfonation for improvement of fragmentation (15) and in combination with $\mathrm{C}$-terminal sequencing methodologies utilizing carboxypeptidase digestion (16). Guanidinated lysine peptides demonstrate increased ionization in both matrix-assisted laser desorption/ionization (MALDI) and electrospray ionization (ESI) mass spectrometry $(6-9,17)$. In short, guanidination has now become an accepted procedure in proteomics laboratories for enhancement of mass spectrometric analysis.

Conservation of a sample has become an increasingly important issue for identification and characterization of low copy proteins in the study of proteomes, despite improvements in instrument sensitivity. For example, liquid chromatography electrospray ionization mass spectrometry (LCESI-MS) modes of investigation typically yield a "one-shot" analysis (i.e., once the sample is injected, it is not usually recovered). In contrast, off- line coupling of the chromatographic step with MALDI-MS allows the LC separation to be "frozen in time" on the MALDI target for subsequent analyses. However, if the peptides of interest are not observed, such circumstances require chemical processing of the sample before repeating the analysis.

There are a limited number of previously published studies in which spotted samples were recovered from the MALDI target for further processing. It has been demonstrated that subsequent analyses (radioimmunoassay and electrophoresis) are possible after sample recovery from the MALDI target (18). Neutral oligosaccharides were recovered and subjected to exoglycosidase treatment for sequencing glycan moieties, but required extensive clean-up steps (19). Peptide sequencing studies by tandem ESI-MS analysis (20) and by derivatization by sulfonation (15) following recovery from the MALDI target have also been reported.

A common drawback exhibited by all of these previously published methods is the necessity for extensive and time-consuming clean-up steps. In this paper, we present procedural details and experimental results of an efficient micro-recovery technique for peptide samples previously spotted onto MALDI targets, requiring only nominal washing of the sample spot. The generation of improved data for peptide mass fingerprint analysis by matrix-assisted laser desorption/ionization time-of-flight mass spectrometry (MALDI-TOF MS) was accomplished by direct derivatization and re-analysis without the need for additional complex clean-up procedures.

\section{MATERIALS AND METHODS}

All materials, including reagents and kits, were sourced from SigmaAldrich (St. Louis, MO, USA), except where noted below. The following proteins were used: apolipoprotein A-I, lysozyme, $\alpha$-lactalbumin, myoglobin, $\beta$-lactoglobulin, fetuin, transferrin, carbonic anhydrase I, and $\beta$-casein.

\section{Sample Preparation}

Proteins were denatured in $8 \mathrm{M}$ 
urea, $20 \mathrm{mM}$ bis-Tris propane, $\mathrm{pH} 8.5$, and reduced with $5 \mathrm{mM}$ tributylphosphine. Alkylation was performed with $15 \mathrm{mM}$ iodoacetamide (ProteoPrep ${ }^{\circledR}$ Reduction and Alkylation kit). Tryptic peptides were prepared either from solution or in-gel digestion using trypsin, proteomics-grade, according to the manufacturer's product literature, as summarized below.

\section{In-Solution Digestion}

To prepare samples for enzymatic digestion, $50 \mathrm{mM}$ ammonium bicarbonate was added to the denatured protein as a diluent to obtain a final urea concentration of $1 \mathrm{M}$. Trypsin was reconstituted in $1 \mathrm{mM} \mathrm{HCl}$ resulting in a final concentration of $1 \mu \mathrm{g} / \mu \mathrm{L}$. A trypsin:protein ratio of 1:50 was achieved by adding $2 \mu \mathrm{g}$ trypsin to 400 $\mu \mathrm{L}$ protein solution $(100 \mu \mathrm{g})$. Digestion was accomplished by overnight incubation at $37^{\circ} \mathrm{C}$.

\section{Electrophoresis and In-Gel Digestion}

Laemmli buffer was added to protein samples followed by heat denaturation for $5 \mathrm{~min}$ at $100^{\circ} \mathrm{C}$. Aliquots $(10 \mu \mathrm{g})$ were loaded on 4\%-20\% Tris-glycine gels (Invitrogen, Carlsbad, CA, USA) in triplicate. The gels were electrophoresed at $150 \mathrm{~V}$ for $1.5 \mathrm{~h}$, then stained with EZBlue ${ }^{\mathrm{TM}}$ stain for $1 \mathrm{~h}$. Gels were destained in Milli- $\mathrm{Q}^{\circledR}$ water (Millipore, Billerica, MA, USA) overnight, followed by proteolysis of excised protein bands using the Trypsin Profile IGD kit, according to the manufacturer's directions.

\section{Mass Spectrometry}

Samples were mixed 1:1 with the matrix solution [ $\alpha$-cyano-4hydroxycinnamic acid, $10 \mathrm{mg} / \mathrm{mL}$ in $70 \%$ acetonitrile $(\mathrm{ACN}), 0.03 \%$ trifluoroacetic acid (TFA)]. Aliquots $(1 \mu \mathrm{L})$ were spotted on the MALDI target and dried under low vacuum. MALDI-TOF mass spectra were acquired using an Axima CFR ${ }^{\mathrm{TM}}$ plus instrument (Shimadzu-Biotech, Manchester, UK) operated in positive ion reflectron mode. Acqui- sition conditions were held consistent for each spectrum to minimize variability, with approximately 100 shots averaged per spectrum. Close external calibration of the mass spectrometer was accomplished using the ProteoMass ${ }^{\mathrm{TM}}$ Peptide MALDI Calibration kit.

\section{Bioinformatics}

Protein identification was accomplished by interrogation of the NCBInr database using the Mascot $^{\circledR}$ search engine at http://www.matrixscience. com. Peak lists were generated consistently by selection of peaks showing an apparent signal-to-noise ratio $\geq 3$. Enzyme selection was trypsin, with $\leq 1$ missed cleavage permitted. Carbamidomethylation of cysteines and, where appropriate, guanidination of lysines were selected as fixed modifications. Taxonomy and protein mass values were unrestricted, and peptide mass tolerance set at $400 \mathrm{ppm}$. Mass values were entered as monoisotopic $\mathrm{MH}+$.
Probability-based molecular weight search (MOWSE) scores are reported.

\section{In-Solution Guanidination}

To serve as reference samples, aliquots of each tryptic peptide solution were guanidinated using the ProteoMass ${ }^{\mathrm{TM}}$ Guanidination kit according to the manufacturer's instructions. Briefly, $10 \mu \mathrm{L}$ samples were incubated with $10 \mu \mathrm{L}$ base reagent $(2.85$ $\mathrm{M}$ ammonium hydroxide) and $10 \mu \mathrm{L}$ $0.30 \mathrm{M} \mathrm{O}$-methylisourea at $65^{\circ} \mathrm{C}$ for 30 $\min$. The reaction was terminated by the addition of $30 \mu \mathrm{L}$ stop solution (10\% TFA). Samples were then combined with matrix solution and deposited on the MALDI target as described above.

\section{Sample Recovery from MALDI Target}

Following acquisition of pre-guanidination mass spectra, the tryptic peptides were recovered by extraction with $70 \%$ ACN. Solubilization was effected by

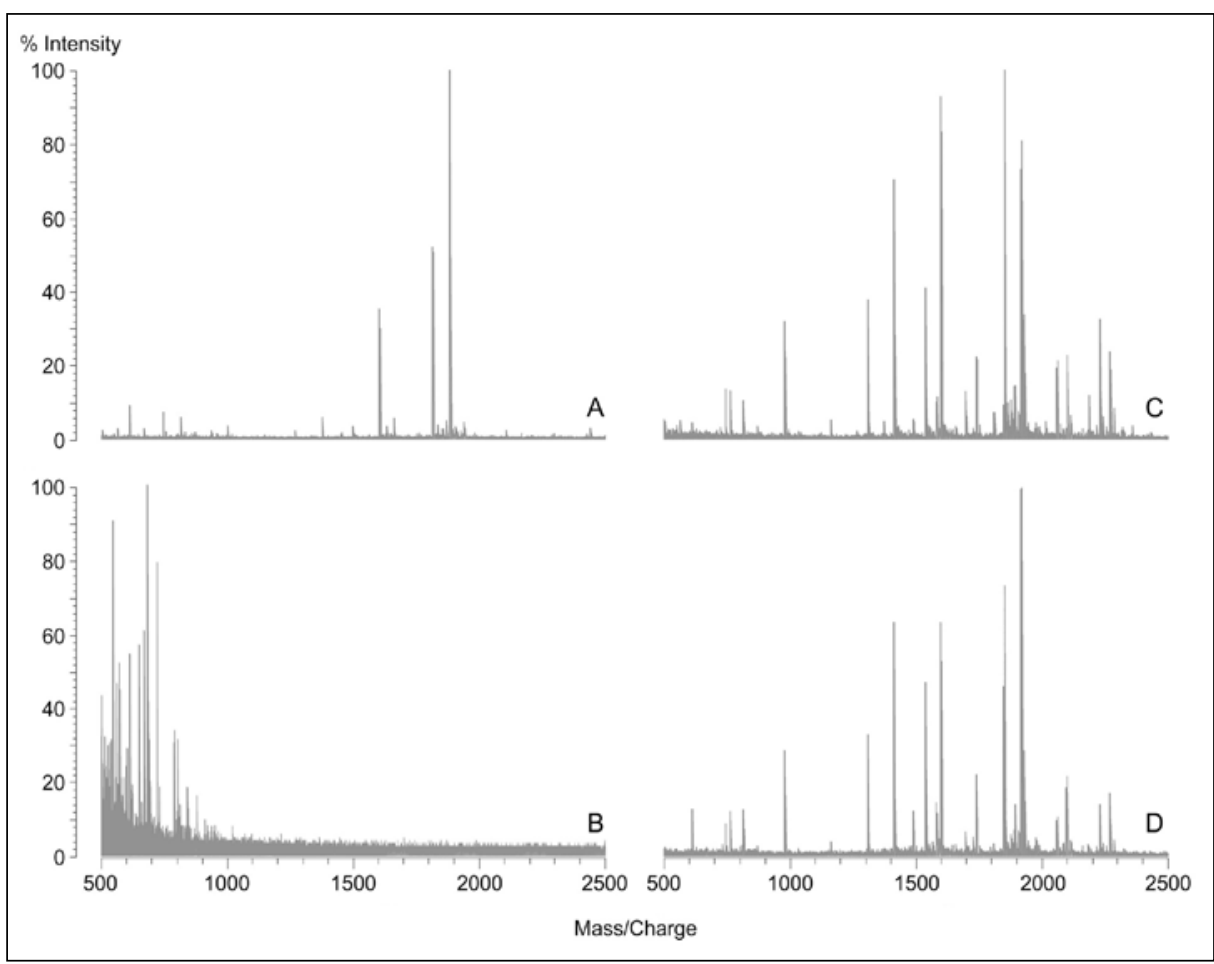

Figure 1. Matrix-assisted laser desorption/ionization time-of-flight mass spectrometry (MALDI-TOF MS) analysis of myoglobin tryptic digest. The peptides were analyzed either (A) without guanidination, (C) with guanidination following the procedure detailed in the ProteoMass Guanidination kit, and (D) with guanidination following efficient micro-recovery of the sample from the target. A greater number of peptides were identified in both guanidinated samples, resulting in an increase in sequence coverage and corresponding higher confidence in protein identification. Spectrum (B) indicates that the peptides were completely removed from the target, in that the peaks observed in this spectrum are consistent with MALDI matrix clusters. 
application of $1 \mu \mathrm{L} 70 \% \mathrm{ACN}$ to the spot, pipeting up and down several times, followed by aspiration into a conical sample tube. This procedure was repeated 2 additional times for a total recovered volume of $3 \mu \mathrm{L}$. All visible sample/matrix spots were completely removed from the MALDI target.

\section{Guanidination of Recovered Peptides}

Recovered peptides were dried in a vacuum centrifuge system, then derivatized by the addition of $5 \mu \mathrm{L} 0.3 \mathrm{M} \mathrm{O}$ methylisourea and $5 \mu \mathrm{L}$ base reagent. Samples were incubated at $65^{\circ} \mathrm{C}$ for 30 min, after which the reaction was then terminated by the addition of $10 \mu \mathrm{L}$ stop solution. The samples were dried in a vacuum centrifuge, and reconstituted in $2 \mu \mathrm{L} \mathrm{70 \%} \mathrm{ACN,} 0.03 \%$ TFA, followed by addition of $1 \mu \mathrm{L}$ matrix solution. A 1$\mu \mathrm{L}$ aliquot was spotted onto the MALDI target, allowed to dry, and washed with $5-\mu \mathrm{L}$ aliquots of $5 \%$ formic acid. MALDI-TOF mass spectra were then re-acquired, as detailed above.

\section{RESULTS AND DISCUSSION}

In this study, guanidination of previously analyzed tryptic peptides was demonstrated, following efficient microrecovery from the MALDI target. As one example, a tryptic digest of myoglobin was analyzed by MALDI-TOF MS (depicted in Figure 1). First, unguanidinated peptides were spotted and analyzed (Figure 1A). The sample was then extracted from the spot with a solution of $70 \%$ ACN, guanidinated, and respotted onto the MALDI target, as described in the Materials and Methods section. The sample was derivatized without the need for involved sample clean-up or separation of the MALDI matrix from the recovered peptides. Analysis of the "residual" sample (Figure 1B), after the re-application of matrix, shows the absence of peptide peaks and indicates complete recovery from the sample spot. The only peaks observed were consistent with the MALDI matrix. For comparison, myoglobin peptides were also guanidinated in solution prior to spotting and analysis (Figure 1C). The spectrum of the micro-recovered and guanidinated sample (Figure 1D) compares favorably to in-solution guanidination. The ability to detect a larger number of peptides can be realized with either modification protocol.

This methodology was also applied to a variety of other model proteins, as depicted in Figure 2. Increases in both sequence coverage and probability of correct protein identification were observed upon utilization of this procedure. When comparing results to samples guanidinated in solution (before mixing with matrix, spotting, and MALDI-TOF analysis), the microrecovery method provides results equivalent to or better than the solution method. Guanidinated samples provided better sequence coverage and data confidence than unguanidinated samples. Improved confidence of protein identification was achieved for species known to be posttranslationally modified and those that are not.

Recovery of intact proteins from MALDI targets has previously demonstrated the viability of proteins for activity assays (10), indicating that sample processing and analysis may not have a detrimental effect upon the analyte. Low sample consumption by MALDI-MS can be used advantageously for recovery of high value samples from the target. This is especially useful in instances where the amount of sample is limited and/ or for samples in which multiple analyses become important for identification or further characterization. Indeed, recovered samples have potential for derivatization by any number of means (21), especially if the original analysis is performed with a shotgun approach as may occur when using
LC-MALDI-MS techniques $(22,23)$. In this situation, samples are "frozen in time," and spots of interest can be recovered, derivatized, and reanalyzed to provide additional information with relative ease.

In conclusion, efficient microrecovery of samples from a MALDI target and their subsequent guanidination provide a fast and convenient method for obtaining additional information from tryptic digests. This is especially relevant to analyses that do not render suitable information upon original inspection or for valuable samples. Utilizing this procedure results in high-quality MALDI spectra, with an associated increase in sequence coverage and enhanced confidence in protein identification and characterization. Furthermore, although tandem mass spectrometry (MS/MS) analysis
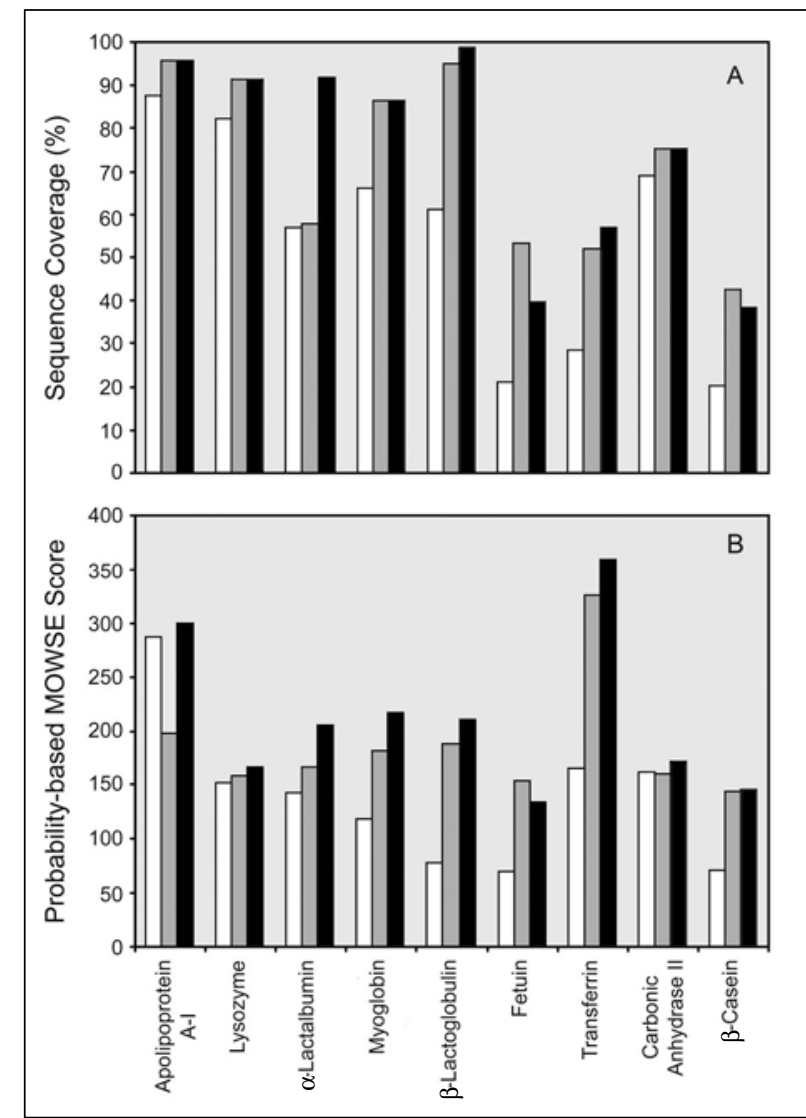

Figure 2. Comparison of sequence coverage (A) and probabilitybased molecular weight search (MOWSE) scores (B) for protein tryptic digests before (white) and after guanidination by both methods. Samples were guanidinated in solution following the procedure for the ProteoMass Guanidination kit directly from a tryptic digest solution (gray) or after efficient micro-recovery of peptide spots from the matrix-assisted laser desorption/ionization (MALDI) target (black). In all cases, increased sequence coverage was noted following guanidination. 
did not form part of this study, it is recognized that sequence query information may potentially be collected from lysine-terminal peptides that were previously of insufficient intensity prior to guanidination.

\section{ACKNOWLEDGMENTS}

The authors wish to acknowledge the assistance of Melissa Spears in preparation of the digest samples. Moreover, we wish to thank the reviewers for their careful reading of the manuscript and helpful suggestions for improvement.

\section{COMPETING INTERESTS STATEMENT}

The authors are all employed by Sigma-Aldrich Corporation, a supplier of a number of products used in this study.

\section{REFERENCES}

1.Krause, E., H. Wenschuh, and P.R. Jungblut. 1999. The dominance of arginine-containing peptides in MALDI-derived tryptic mass fingerprints of proteins. Anal. Chem. 71:4160-4165.

2.Baumgart, S., Y. Lindner, R. Kühne, A. Oberemm, H. Wenschuh, and E. Krause. 2004. The contributions of specific amino acid side chains to signal intensities of peptides in matrix-assisted laser desorption/ionization mass spectrometry. Rapid Commun. Mass Spectrom. 18:863-868.

3.Zhu, Y.F., K.L. Lee, K. Tang, S.L. Allman, N.I. Taranenko, and C.H. Chen. 1995. Revisit of MALDI for small proteins. Rapid Commun. Mass Spectrom. 9:1315-1320.

4.Wenschuh, H., P. Halada, S. Lamer, P. Jungblut, and E. Krause. 1998. The ease of peptide detection by matrix-assisted laser desorption/ionization mass spectrometry: the effect of secondary structure on signal intensity. Rapid Commun. Mass Spectrom. 12:115-119.

5.Kratzer, R., C. Eckerskorn, M. Karas, and F. Lottspeich. 1998 Suppression effects in enzymatic peptide ladder sequencing using ultraviolet-matrix assisted laser desorption/ ionization-mass spectrometry. Electrophoresis 19:1910-1919.

6.Beardsley, R.L., J.A. Karty, and J.P. Reilly. 2000. Enhancing the intensities of lysine-terminated tryptic peptide ions in matrix-assisted laser desorption/ionization mass spectrometry. Rapid Commun. Mass Spectrom. 14:21472153.

7.Hale, J.E., J.P. Butler, M.D. Knierman, and G.W. Becker. 2000. Increased sensitivity of tryptic peptide detection by MALDI-TOF mass spectrometry is achieved by conversion of lysine to homoarginine. Anal. Biochem. 287:110-117.

8.Brancia, F.L., S.G. Oliver, and S.J. Gaskell. 2000. Improved matrix-assisted laser desorption/ionization mass spectrometric analysis of tryptic hydrolysates of proteins following guanidination of lysine-containing peptides. Rapid Commun. Mass Spectrom. 14:20702073.

9.Beardsley, R.L. and J.P. Reilly. 2002. Optimization of guanidination procedures for MALDI mass mapping. Anal. Chem. 74:18841890.

10.Thevis, M., R.R. Ogorzalek Loo, and J.A. Loo. 2003. In-gel derivatization of proteins for cysteine-specific cleavages and their analysis by mass spectrometry. J. Proteome Res. 2:163-172.

11.Fenaille, F., F. Morgan, V. Parisod, J.C. Tabet, and P.A. Guy. 2004. Solid-state glycation of $\beta$-lactoglobulin by lactose and galactose: localization of the modified amino acids using mass spectrometric techniques. J. Mass. Spectrom. 39:16-28.

12.Cagney, C. and A. Emili. 2002. De novo peptide sequencing and quantitative profiling of complex protein mixtures using mass-coded abundance tagging. Nat. Biotechnol. 20:163170 .

13.Lemmel, C., S. Weik, U. Eberle, J. Dengjel, T. Kratt, H.-D. Becker, H.-G. Rammensee, and S. Stevanovic. 2004. Differential quantitative analysis of MHC ligands by mass spectrometry using stable isotope labeling. Nat. Biotechnol. 22:450-454.

14.Brancia, F.L., H. Montgomery, K. Tanaka, and S. Kumashiro. 2004. Guanidino labeling derivatization strategy for global characterization of peptide mixtures by liquid chromatography matrix-assisted laser desorption/ ionization mass spectrometry. Anal. Chem. 76:2748-2755

15.Keough, T., M.P. Lacey, and R.S. Youngquist. 2002. Solid-phase derivatization of tryptic peptides for rapid protein identification by matrix-assisted laser desorption/ionization mass spectrometry. Rapid Commun. Mass Spectrom. 16:1003-1015.

16.Bonetto, V., A.C. Bergman, H. Jörnvall, and R. Sillard. 1997. C-terminal sequence analysis of peptides using carboxypeptidases and mass spectrometry after derivatization of Lys and Cys residues. Anal. Chem. 69:13151319.

17.Brancia, F.L., M.E. Openshaw, and S. Kumashiro. 2002. Investigation of the electrospray response of lysine-, arginine-, and homoarginine-terminal peptide mixtures by liquid chromatography/mass spectrometry. Rapid Commun. Mass Spectrom. 16:22552259.

18.Cerpa-Poljak, A., A. Jenkins, and M.W. Duncan. 1995. Recovery of peptides and proteins following matrix-assisted laser desorption/ionization mass spectrometry. Rapid Commun. Mass Spectrom. 9:233-239.

19.Küster, B., T.J.P. Naven, and D.J. Harvey. 1996. Rapid approach for sequencing neutral oligosaccharides by exoglycosidase digestion and matrix-assisted laser desorption/ioniza- tion time-of-flight mass spectrometry. J. Mass Spectrom. 31:1131-1140.

20.Terry, D.E., E. Umstot, and D.M. Desiderio. 2004. Optimized sample-processing time and peptide recovery for the mass spectrometric analysis of protein digests. J. Am. Soc. Mass Spectrom. 15:784-794.

21.Rosenfeld, J.M. 2003. Derivatization in the current practice of analytical chemistry. Trends Anal. Chem. 22:785-798.

22.Zhen, Y., N. Xu, B. Richardson, R. Becklin, J.R. Savage, K. Blake, and J.M. Peltier. 2004. Development of an LC-MALDI method for the analysis of protein complexes. J. Am. Soc. Mass Spectrom. 15:803-822

23.Bodnar, W.M., R.K. Blackburn, J.M. Krise, and M.A. Moseley. 2003. Exploiting the complementary nature of LC/MALDI/MS/ MS and LC/ESI/MS/MS for increased proteome coverage. J. Am. Soc. Mass Spectrom. 14:971-979.

Received 12 August 2004; accepted 30 September 2004.

Address correspondence to Graham B.I. Scott, Sigma-Aldrich Corporation, P.O. Box 14508, St. Louis, MO 63178, USA. e-mail: gscott@sial.com 